\title{
Innate Immune Activation and Circulating Inflammatory Markers in Preschool Children
}

\begin{abstract}
Fiona Collier ${ }^{1,2 *}$, Cerys Chau ${ }^{3}$, Toby Mansell ${ }^{3}$, Keshav Faye-Chauhan ${ }^{1}$, Peter Vuillermin 1,2,3, Anne-Louise Ponsonby ${ }^{1,4}$, Richard Saffery ${ }^{3,4}$, Mimi L. K. Tang ${ }^{3,5}$, Martin O'Hely ${ }^{1,2,3}$, John Carlin ${ }^{3,5}$, Lawrence E. K. Gray ${ }^{2}$, Siroon Bekkering ${ }^{3,6 t}$, David Burgner ${ }^{3,5,7 t}$ and the Barwon Infant Study Investigator Group ${ }^{\ddagger}$
\end{abstract}

1 School of Medicine, Deakin University, Geelong, VIC, Australia, ${ }^{2}$ Child Health Research Unit, Barwon Health, Geelong, VIC, Australia, ${ }^{3}$ Murdoch Children's Research Institute, Royal Children's Hospital, Parkville, VIC, Australia, ${ }^{4}$ Department of Neuroepidemiology, The Florey Institute of Neuroscience and Mental Health, Parkville, VIC, Australia, ${ }^{5}$ Department of Paediatrics, Melbourne University, Parkville, VIC, Australia, ${ }^{6}$ Department of Internal Medicine and Radboud Institute for Molecular Life Science (RIMLS), Radboud University Medical Center, Nijmegen, Netherlands, ${ }^{7}$ Department of Paediatrics, Monash University, Clayton, VIC, Australia

Early childhood is characterised by repeated infectious exposures that result in inflammatory responses by the innate immune system. In addition, this inflammatory response to infection is thought to contribute to the epidemiological evidence linking childhood infection and adult non-communicable diseases. Consequently, the relationship between innate immune responses and inflammation during early life may inform prevention of NCDs later in life. In adults, non-genetic host factors such as age, sex, and obesity, strongly impact cytokine production and circulating mediators, but data in children are lacking. Here, we assessed cytokine responses and inflammatory markers in a population of healthy preschool children (mean age 4.2 years). We studied associations between cytokines, plasma inflammatory markers and non-genetic host factors, such as sex, age, adiposity, season, and immune cell composition. Similar to adults, boys had a higher inflammatory response than girls, with IL-12p70 and IL-10 upregulated following TLR stimulation. Adiposity and winter season were associated with increased circulating inflammatory markers but not cytokine production. The inflammatory markers GlycA and hsCRP were positively associated with production of a number of cytokines and may therefore reflect innate immune function and inflammatory potential. This dataset will be informative for future prospective studies relating immune parameters to preclinical childhood NCD phenotypes.

Keywords: innate immune activation, cytokines, preschool children, human functional genomics, systemic inflammation

\section{INTRODUCTION}

Non-communicable diseases (NCDs) result in huge and increasing human and economic costs globally. Development of innovative and effective prevention of NCDs requires better understanding of modifiable pathogenic pathways across the life course (1). NCDs generally manifest in adulthood and disproportionately affect males $(2,3)$. The end-organ damage manifests 
as a range of conditions and inflammation is the central pathogenic process (4). The trajectories that lead to chronic inflammation begin in early life (5). Understanding the variation and early determinants of inflammatory responses in healthy children may therefore highlight opportunities for NCD prevention.

Early childhood is characterised by repeated infectious exposures that result in inflammatory responses by the innate immune system $(6,7)$. The intensity of these responses in part determines the clinical severity of infection and varies markedly between individuals (8). This inflammatory response to infection is thought to contribute to the epidemiological evidence linking childhood infection and adult NCDs (9). Cytokines mediate these inflammatory responses, but to date, investigation of variation in cytokine production capacity in the general population has been limited to adults (10).

The toll-like receptor (TLR) pathway is central to innate immune responses to microbial stimuli (11) and a potential therapeutic target for inflammatory diseases (12). There are extensive data on TLR responses in older children and adults $(13,14)$, but few analogous data in preschool children, the age group with the highest incidence of infection (15). In particular, it is unclear whether variation in TLR responses with sex and environmental exposures reported in adults $(16,17)$ is also evident in early childhood. It is also unknown whether inflammatory markers, such as glycoprotein acetyls (GlycA), an NMR-derived marker of cumulative inflammation (18-20) and high sensitivity C-reactive protein (hsCRP) correlate with innate immune responses.

In the current study, we aimed to investigate innate immune responses to two key bacterial TLR ligands (lipopolysaccharide, LPS; and peptidoglycan, PGN) in a cohort of healthy preschool children, and relate these responses to GlycA and hsCRP. We also examined how host and environmental factors (sex, age, adiposity, granulocyte/monocyte proportions, or season) were related to cytokine production and circulating inflammatory markers.

\section{METHODS}

\section{Study Cohort}

Children in this study were participants of the Barwon Infant Study (BIS), which recruited pregnant mothers through antenatal clinics at two major hospitals in Geelong (Victoria, Australia) between 2010-2013 (20). The final inception cohort included 1,064 mothers with 1,074 infants (10 twin pairs). Children who visited the BIS for their preschool clinical review (age 3.9-5.6 years) were asked to complete a questionnaire, undergo a skin-prick allergy test (SPT) and provide a blood sample. The cohort used in this study was a selection of subjects that had both a TLR stimulation assay and relevant metadata. Ethics approval for BIS (Project number 10/24) was obtained through the Barwon Health Human Research Ethics Committee (HREC).

\section{Data Collection}

At the preschool clinical review, parents were asked to complete a survey including questions regarding their child's current wellbeing, health and any recent illness or temperature. Children also had their body weight [measured with bioelectrical impedance analysis (BIA) scale (Tanita, Kewdale, Australia, model BC-420MA)], height [measuring rod stadiometer (Seca, Hamburg, Germany, model no. 213)] and waist circumference (tape measure) recorded. BMI for each participant was calculated by dividing weight by height in metres squared $\left(\mathrm{kg} / \mathrm{m}^{2}\right)$. Measurement of body fat were obtained using the BIA scale.

\section{Blood Sampling and Innate Immune Assays}

Blood samples were collected in preservative-free sodium heparinised tubes. To quantify cytokine responses of immune cells under conditions similar to those in vivo, and to maximise outputs from small paediatric blood volumes, we measured the production of monocyte-derived cytokines following stimulation of whole blood. Precisely two hours from blood collection, an aliquot of whole blood was diluted 1:2 with RPMI 1640 growth medium and transferred to plate strips that consist of 8 wells (the size of a 96 well plate) each containing $20 \mu \mathrm{L}$ of either RPMI (growth medium), the gram-negative bacterial membrane component, lipopolysaccharide (LPS, $100 \mathrm{ng} / \mathrm{mL}$ final concentration) or gram-positive bacterial membrane component, peptidoglycan (PGN, 10ug/mL). These represent either unstimulated, TLR4, or TLR2-stimulated conditions respectively. $180 \mu \mathrm{L}$ of diluted blood was added to each well and cells were stimulated for 24 hours at $37^{\circ} \mathrm{C}$ in $5 \% \mathrm{CO}_{2}$. To reduce evaporation, additional plate strips containing only water were incubated alongside the experimental strips. Following the 24-hour incubation, the strips were centrifuged to pellet the blood cells, and the supernatant collected in two $50 \mu \mathrm{L}$ aliquots to be stored at $-80^{\circ} \mathrm{C}$ until cytokine analysis.

\section{Flow Cytometry}

Following phlebotomy, a separate aliquot of $100 \mu \mathrm{L}$ whole blood underwent flow cytometry (Becton Dickinson (BD) FACSCanto ${ }^{\mathrm{TM}}$ ) to identify proportions of target cell populations including monocytes and granulocytes, expressed as the percentage of total white blood cells (WBC). WBC were stained with (i) anti-human CD4-FITC, anti-human CD3-PE and antihuman CD45-PerCP; or (ii) anti-human CD14-FITC, anti-human CD16-PE and anti-human HLA-DR-PECy5, before red cell lysis and formalin fixation. Monocytes and granulocytes were discriminated based on side scatter (SSC) and CD45 expression (21). The proportion of activated nonclassical monocytes $\left(\mathrm{CD} 14^{+} /\right.$ $\mathrm{CD} 16^{++}$, as \% of total monocytes) was also determined by gating to the HLA-DR ${ }^{+}$monocytes. All antibodies were sourced from BD Biosciences (San Jose, California, US).

\section{Cytokine Quantification}

Cytokines were quantified using the Bio-Rad Bio-Plex Pro ${ }^{\text {TM }}$ cytokine assay kit and detection software, with small modifications to manufacturer instructions. Standards were prepared in a dilution series using the standard kit-provided diluent. Frozen samples were thawed and added to plates that contained 8 standards in duplicate (including blank), and inter- 
plate controls. Manual steps in the assay included addition of magnetic beads, detection antibodies and streptavidinphycoerythrin dye, with washing and incubation in between each step. Plates were analysed using the xPONENT MAGPIX ${ }^{\circledR}$ instrument (Bio-Rad ${ }^{\circledR}$ ). Standard curves were produced based on the standard dilutions and optimised for a recovery rate of 70 $130 \%$. Final measures in pg/mL for IL-1 $\beta$, IL-6, TNF $\alpha$, IL- $12 p 70$ (pro-inflammatory), IL-1RA and IL-10 (anti-inflammatory) were derived by the Bio-Plex Manager ${ }^{\mathrm{TM}}$ Software using the standard curves. Cytokine concentrations above the standard curve's upper limit of quantification (ULOQ) were excluded whereas cytokine concentrations below the lower level of quantification (LLOQ) were replaced with a value equal to $50 \%$ of LLOQ.

\section{GlycA and hsCRP Quantification}

Markers of inflammation (GlycA and hsCRP) were measured in plasma samples from the bloods collected in sodium heparin tubes. High-throughput proton NMR metabolomics (Nightingale Health, Helsinki, Finland) quantified GlycA (mmol/L) (22), and hsCRP (ug/ml) was determined using ELISA Human C-Reactive Protein/CRP assay (R\&D systems, DY1707). Both measures were log-transformed (base 10), and CRP measurements equal to zero $(n=47)$ were assigned a value equal to $50 \%$ of the lowest measure $(0.001 \mathrm{ug} / \mathrm{ml})$.

\section{Statistical Analysis}

Cytokine measures were log transformed (base 10). BMI z-score standardised for age and sex (based on WHO Child Growth Charts (age $<5$ years) and the WHO Reference 2007 (age $>=5$ years) was calculated using the zanthro function from the dm0004_1 userdeveloped Stata package (23). Spearman correlations were used to quantify the magnitude and direction of association between the cytokines with the coefficient $\left(r_{s}\right)$ given for each pair of cytokines. Univariable linear regression was used to investigate the associations between exposures, including sex, age, adiposity and innate immune cell proportions, and cytokine levels (log transformed) in the three conditions (unstimulated, and following stimulation with LPS and PGN), as well as the inflammatory markers. The p-values are presented unadjusted for multiple comparisons. Directed acyclic graphs (http://www.dagitty. net) were constructed to determine the minimal sufficient adjustment set for estimating the total effect of the inflammatory markers (GlycA or hsCRP) on the stimulated cytokine levels (Supplementary Figure 4). Sensitivity analyses were performed by excluding children with hsCRP above $5 \mathrm{ug} / \mathrm{ml}$, which may be indicative of an active infection $(24,25)$. All statistical analysis and graphics were completed using Stata Statistical Software: Release 15 (College Station, TX: StataCorp LLC).

\section{RESULTS}

\section{Baseline Host and Environmental Characteristics}

Complete baseline characteristics of the cohort are described elsewhere (20). We have presented the most important baseline characteristics for this study in Supplementary Table 1 according to sex. 285 participants were included in this study, with $52 \%$ male. The mean age for both sexes was 4.2 years. Bloods were collected across all seasons of the year. Mean weight and fat mass was $17.9 \mathrm{~kg}$ (range $12.6-34.4 \mathrm{~kg}$ ) and $19.7 \%$ (range 10.2-29.0\%), respectively. Thirty-three children (14.2\%) were classified as overweight (BMI Z-score $>1 \mathrm{SD}$ ), and 5 children (1.9\%) as obese (BMI z-score $>2$ SD) and boys had slightly higher height, weight, BMI and fat mass. Monocyte proportions, but not

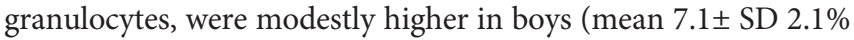
compared to $6.5 \pm 1.9 \%$ in girls) whereas the proportion of activated nonclassical monocytes in boys was lower (median 5.4 (IQR 3.4-8.1)\% compared to 6.5 (4.2-10.2)\%). hsCRP levels were overall lower in males, however $9(6.1 \%)$ boys and $3(2.1 \%)$ girls had hsCRP levels above $5 \mathrm{ug} / \mathrm{ml}$ possibly indicative of active infection.

\section{Circulating Inflammatory Markers (GlycA and hsCRP) Associate With Host- and Environmental Factors}

Following $\log$ transformation of the inflammatory markers, levels of GlycA were only moderately correlated with hsCRP $(r=0.41$, Supplementary Figure 1). Levels of hsCRP were lower in boys (Supplementary Table 1, Table 1), and GlycA and hsCRP were both positively associated with BMI z-score (Table 1). In addition, levels of both inflammatory markers were higher in winter (Supplementary Figure 2, Table 1), and were associated with the proportion of granulocytes (Table 1), indicating overall increased inflammatory status.

\section{TLR-Stimulation and Monocyte Derived Cytokines}

To quantify cytokine responses of immune cells under conditions similar to those in vivo, and to maximise outputs from small paediatric blood volumes, we measured the production of monocyte-derived cytokines after stimulation of whole blood, rather than purified cell populations. Cells were stimulated for 24 hours using either gram-negative or grampositive bacterial ligands.

\section{Cytokine Correlations}

Pro-inflammatory and anti-inflammatory monocyte-derived cytokines (IL-1 $\beta$, IL-6, TNF $\alpha$, IL-12p70, IL-1RA, IL-10) were measured following stimulation of TLR4 (LPS) and TLR2 (PGN) in whole blood (Supplementary Table 2). Unstimulated cytokine levels moderately predicted the TLR-stimulated levels (except for IL-1 $\beta$ in response to LPS) (Supplementary Figure 3). Overall, intra-individual cytokine production in unstimulated and following stimulation by each TLR ligand were modestly to strongly correlated ( $r_{s}$ range $\left.0.23-0.84\right)$ (Supplementary Figure 4).

\section{Associations of Cytokines With Host and Environmental Factors}

Both pro- and anti-inflammatory cytokine production following TLR stimulation was higher in boys, particularly for IL-12p70 and IL-10 after LPS stimulation, and IL-6, IL-1RA, IL-12p70 and IL-10 following PGN stimulation (Figure 1, Table 2). Following adjustment for sex, IL-1 $\beta$ was correlated with age in the 
TABLE 1 | Associations between inflammatory factors and sex, child age, adiposity (BMl and \%fat mass), seasons and innate immune cell proportions.

\begin{tabular}{|c|c|c|c|c|c|c|c|c|}
\hline & & $\begin{array}{l}\text { Adjusted } \\
\text { for }\end{array}$ & $\begin{array}{c}\text { GlycA } \\
\text { Log10 } \\
(\mathrm{mmol} / \mathrm{L})\end{array}$ & $95 \% \mathrm{Cl}$ & p-value & $\begin{array}{l}\text { hsCRP } \\
\text { Log10(ug/ } \\
\text { ml) }\end{array}$ & $95 \% \mathrm{Cl}$ & p-value \\
\hline \multicolumn{2}{|l|}{$\begin{array}{l}\text { Sex (compared to } \\
\text { female) }\end{array}$} & & $-0,01$ & $(-0.02,0.01)$ & 0,285 & $-0,33$ & & \\
\hline \multicolumn{2}{|l|}{$\begin{array}{l}\text { Log10 } \\
\text { (Age, yr) }\end{array}$} & Sex & 0,03 & $(-0.21,0.26)$ & 0,820 & $-0,30$ & & \\
\hline \multicolumn{2}{|l|}{ BMI z-score } & & 0,01 & $(0.00,0.02)$ & 0,010 & 0,16 & & \\
\hline \multirow[t]{2}{*}{$\begin{array}{l}\text { Log10 } \\
\text { (\%Fat Mass) }\end{array}$} & & Sex, Age & 0,19 & $(-0.01,0.39)$ & 0,061 & 4,52 & & \\
\hline & Summer & & $-0,03$ & $(-0.05,-0.02)$ & $<0.0001$ & $-0,34$ & & \\
\hline \multirow{3}{*}{$\begin{array}{l}\text { Season (compared to } \\
\text { Winter) }\end{array}$} & Autumn & & $-0,02$ & $(-0.03,0.00)$ & 0,035 & $-0,63$ & & \\
\hline & Spring & & 0,02 & $(-0.04,0.00)$ & 0,017 & $-0,38$ & & \\
\hline & Granulocytes (\% of WB) & & 0,14 & $(0.08,0.21)$ & $<0.0001$ & 3,85 & & \\
\hline \multirow{2}{*}{$\begin{array}{l}\text { Innate Immune } \\
\text { Populations }\end{array}$} & Log10(Monocytes, \% of WB) & Sex, Age & $-0,03$ & $(-0.08,0.02)$ & 0,684 & 0,24 & & \\
\hline & $\begin{array}{l}\text { Log10 } \\
\text { (activated nonclassical Monocytes, \% of total } \\
\text { monocytes) }\end{array}$ & & 0,00 & $(-0.02,0.02)$ & 0,730 & 0,65 & & \\
\hline
\end{tabular}

Linear regression analysis, *denotes adjusted for sex. Beta coefficients (units=Log10(mmol/L GlycA or ug/ml hsCRP) per variable) with $95 \%$ confidence interval (Cl) and $p$ value. The strength of association is denoted by either shades of blue (negative) or red (positive).

unstimulated and LPS stimulated group (Table 2). There was no evidence that adiposity was associated with cytokine responses (Table 2). Season of blood collection was also not associated with cytokine production (data not shown).

TNF $\alpha$ levels were positively associated with the percentage of monocytes in the stimulated conditions (adjusted for sex, Supplementary Table 3). IL-12p70 was associated with the proportion of monocytes in unstimulated blood and following PGN stimulation (Supplementary Table 3). IL-1RA was associated with the proportion of granulocytes in unstimulated and TLR-stimulated blood (Supplementary Table 3) and there was weaker evidence that IL-6 was associated with granulocyte proportion in unstimulated blood (Supplementary Table 3).

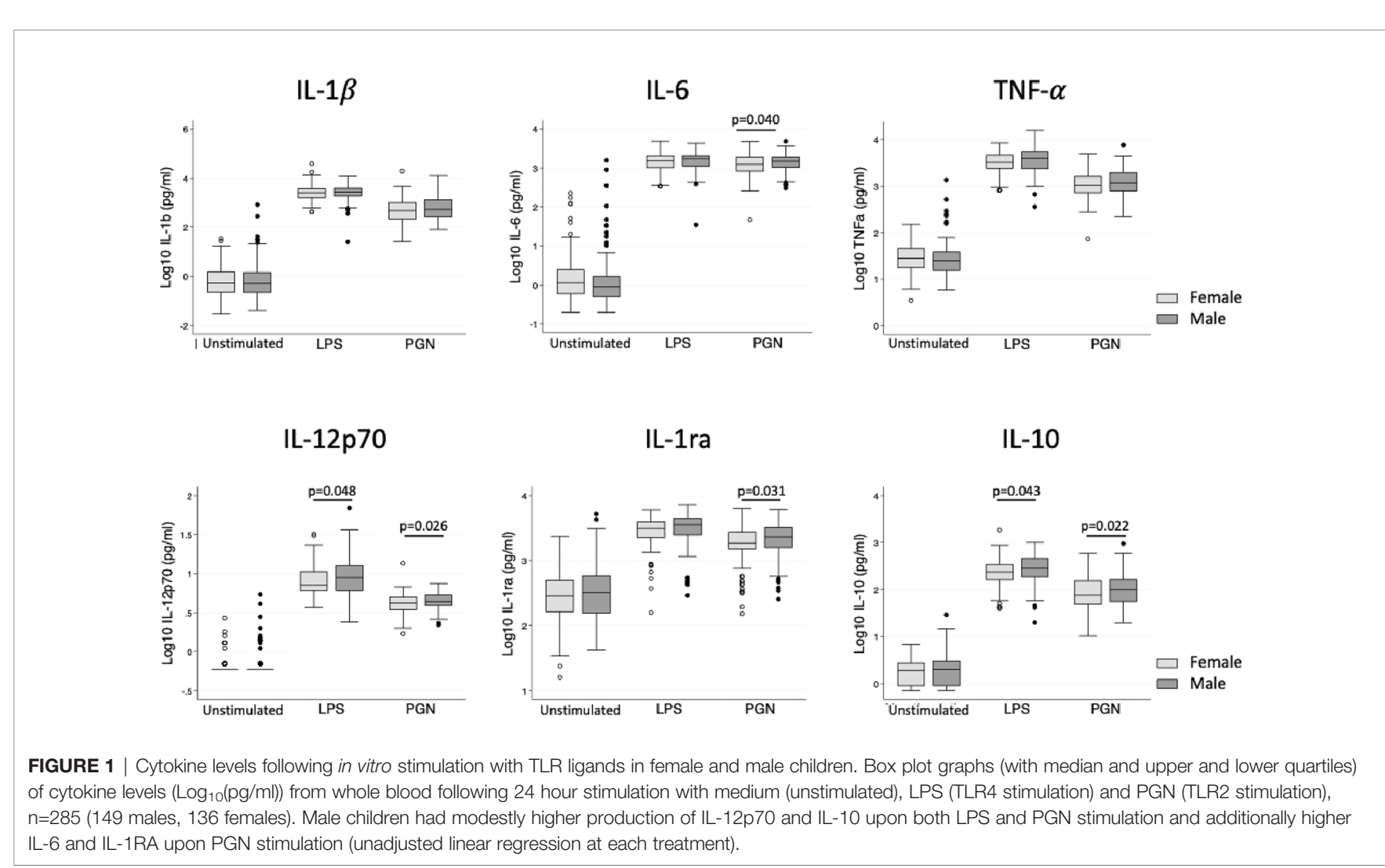


TABLE 2 | Association between sex, child age, and adiposity, and log transformed cytokine levels under unstimulated and stimulated conditions.

\begin{tabular}{|c|c|c|c|c|c|}
\hline & Stimulation Conditions & $\begin{array}{c}\text { Sex } \\
\text { Compared to Female }\end{array}$ & $\begin{array}{l}\log _{10}(\text { Age, } y r) \\
\text { Adjusted for Sex }\end{array}$ & BMI z-score & $\begin{array}{c}\log _{10}(\% \text { Fat Mass }) \\
\text { Adjusted for Age and Sex }\end{array}$ \\
\hline \multirow[t]{3}{*}{ IL-1 $\beta$} & Unstimulated & $-0.00(-0.16,0.15)$ & $0.30(0.02,0.57)$ & $0.07(-0.01,0.16)$ & $0.01(-0.01,0.04)$ \\
\hline & LPS & $0.00(-0.08,0.08)$ & $-0.18(-0.33,-0.04)$ & $-0.03(-0.07,0.02)$ & $-0.01(-0.02,0.01)$ \\
\hline & PGN & $0.07(-0.03,0.18)$ & $-0.06(-0.25,0.13)$ & $-0.00(-0.03,0.01)$ & $-0.00(-0.02,0.01)$ \\
\hline \multirow[t]{3}{*}{ IL-6 } & Unstimulated & $-0.07(-0.22,0.08)$ & $0.18(-0.09,0.44)$ & $0.01(-0.07,0.09)$ & $0.00(-0.02,0.03)$ \\
\hline & LPS & $0.02(-0.03,0.08)$ & $-0.04(-0.15,0.06)$ & $0.01(-0.02,0.04)$ & $0.01(-0.00,0.02)$ \\
\hline & PGN & $0.06(0.00,0.12)$ & $-0.07(-0.17,0.04)$ & $0.02(-0.02,0.05)$ & $0.00(-0.01,0.01)$ \\
\hline \multirow[t]{3}{*}{ TNF $\alpha$} & Unstimulated & $-0.01(-0.09,0.07)$ & $0.09(-0.05,0.23)$ & $0.01(-0.04,0.05)$ & $0.00(-0.01,0.02)$ \\
\hline & LPS & $0.05(-0.01,0.11)$ & $0.00(-0.11,0.11)$ & $-0.01(-0.04,0.02)$ & $0.00(-0.01,0.01)$ \\
\hline & PGN & $0.06(-0.01,0.12)$ & $0.00(-0.12,0.12)$ & $-0.02(-0.05,0.02)$ & $-0.01(-0.02,0.01)$ \\
\hline \multirow[t]{3}{*}{ IL-12p70 } & Unstimulated & $0.02(-0.01,0.05)$ & $0.04(-0.01,0.09)$ & $0.00(-0.01,0.02)$ & $0.00(-0.00,0.01)$ \\
\hline & LPS & $0.05(0.00,0.10)$ & $-0.02(0.12,0.07)$ & $-0.01(-0.04,0.02)$ & $0.00(-0.01,0.01)$ \\
\hline & PGN & $0.03(0.00,0.06)$ & $-0.00(-0.05,0.05)$ & $-0.01(-0.02,0.01)$ & $-0.00(-0.01,0.00)$ \\
\hline \multirow[t]{3}{*}{ IL-1RA } & Unstimulated & $0.04(-0.05,0.14)$ & $0.13(-0.02,0.30)$ & $0.03(-0.01,0.08)$ & $0.00(-0.01,0.02)$ \\
\hline & LPS & $0.05(-0.01,0.10)$ & $-0.04(-0.14,0.07)$ & $0.02(-0.01,0.05)$ & $0.01(-0.00,0.02)$ \\
\hline & PGN & $0.07(0.01,0.13)$ & $-0.01(-0.12,0.10)$ & $0.01(-0.02,0.04)$ & $-0.00(-0.01,0.01)$ \\
\hline \multirow[t]{3}{*}{ IL-10 } & Unstimulated & $0.02(-0.05,0.08)$ & $0.04(-0.08,0.16)$ & $0.02(-0.02,0.06)$ & $-0.00(-0.01,0.01)$ \\
\hline & LPS & $0.07(0.00,0.14)$ & $0.04(-0.08,0.16)$ & $0.02(-0.02,0.05)$ & $0.00(-0.01,0.01)$ \\
\hline & PGN & $0.09(0.01,0.17)$ & $-0.13(-0.27,0.00)$ & $-0.01(-0.05,0.03)$ & $0.00(-0.01,0.01)$ \\
\hline
\end{tabular}

Linear regression analysis. Beta coefficients (units $=\log _{10}(\mathrm{pg} / \mathrm{ml}$ of cytokine) per variable) with $95 \%$ confidence interval (Cl). Those in bold indicate a $p$ value $<0.05$

\section{Relationships Between Circulating Inflammatory Markers and TLR-Stimulated Monocyte-Derived Cytokines}

We next investigated associations between circulating inflammatory markers GlycA and CRP and cytokine levels in regression models adjusted for age and sex. There was evidence that both unstimulated and TLR-stimulated cytokine levels were positively associated with GlycA and hsCRP. GlycA was positively associated with the pro-inflammatory cytokines IL- $1 \beta$ and IL- 6 in the unstimulated and PGN stimulated group (Figure 2A), and with the anti-inflammatory cytokines IL-1RA and IL-10 positively associated with GlycA in the LPS and PGN stimulated groups (Figure 2A). High sensitivity CRP was associated with IL-1 $\beta$ and IL-6 in unstimulated blood, with IL-6 following LPS and PGN stimulation (Figure 2B), and with IL-1RA and IL-10 following LPS and PGN stimulation (Figure 2B).

Sensitivity analyses were performed by excluding children with hsCRP > $5 \mathrm{ug} / \mathrm{ml}$, levels possibly indicative of recent or active infection. The associations observed between the circulating inflammatory markers and cytokines in
A

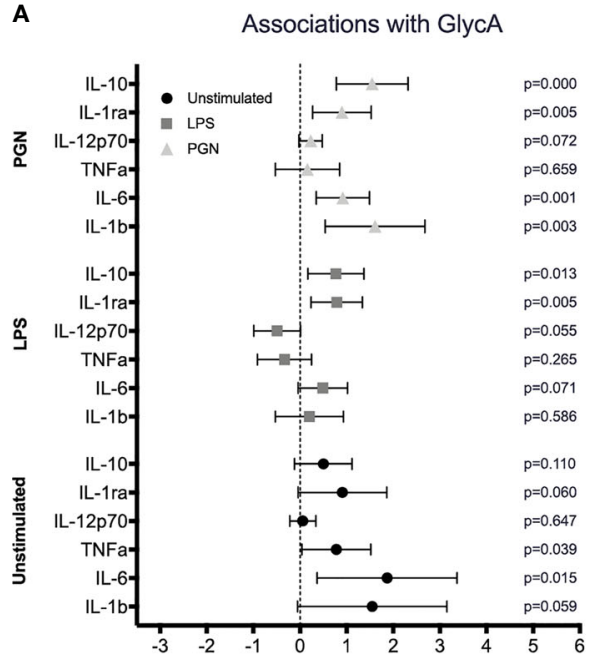

Mean difference in $\log _{10}$ cytokine levels (coefficient $\pm 95 \% \mathrm{Cl}$ )
B

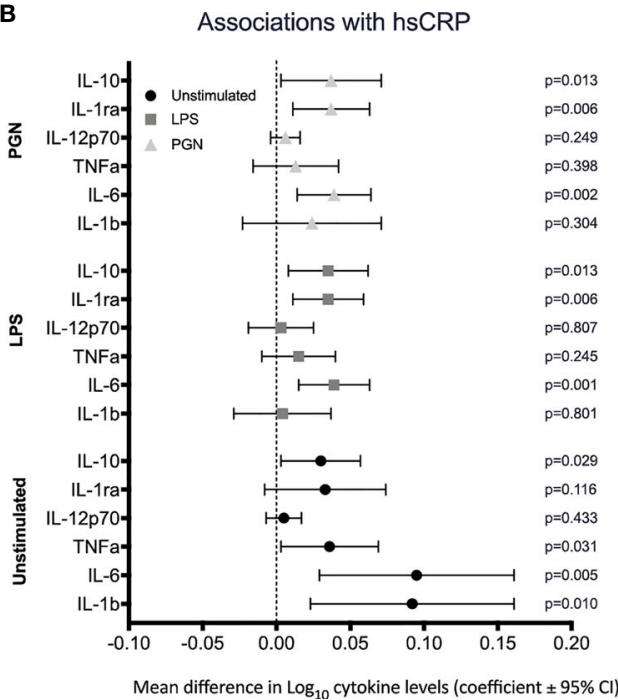

FIGURE 2 | Association between circulating inflammatory markers GlycA and hsCRP and cytokine levels. Cytokines were quantified following stimulation of whole blood in either media (unstimulated) or TLR ligands, LPS and PGN. The estimated mean differences in cytokine levels (Log $10(\mathrm{pg} / \mathrm{mll}))$ were calculated per 10 -fold difference in (A) GlycA, $\log _{10}(\mathrm{mmol} / \mathrm{L})$ and $(\mathbf{B}) \mathrm{hsCRP}, \log _{10}(\mathrm{\mu g} / \mathrm{ml})$. Linear regression analyses of cytokine level on inflammatory marker adjusted for sex, age and innate immune cell populations (as determined a priori). 
unstimulated and stimulated conditions persisted but were modestly attenuated (Supplementary Figures 4A, B).

\section{DISCUSSION}

Here we present the largest study to date investigating the relationships between cytokine responses following TLR stimulation in young children and circulating inflammatory markers. The findings provide novel insights into innate immune activity in healthy preschool children and will inform future studies into early onset of inflammatory diseases in this and other cohorts. Knowledge on healthy innate immune responses provides opportunities for comparison in other cohorts with children or young adults at risk for or with NCDs. Furthermore, the children in our cohort are being followed longitudinally, and further innate immune and CVD related measures are to be performed at 8-10 years of age. In this study, levels of cytokines, both unstimulated and TLRstimulated, were broadly correlated with each other, and with sex, age and innate immune cell populations, but not with BMI or adiposity measures. Circulating inflammatory markers were associated with cytokine production following TLR stimulation, independent of recent infection.

Infections, which are common in early childhood (15), are associated with increased risk of later NCDs $(3,26,27)$. In early childhood, the innate immune responses are central to the defence against infection and are characterised by inflammatory responses (7). Variation in these immune responses in adults is extensively reported $(10,28)$, but data regarding innate immune capacity and inflammation in preschool children are scarce. In our cohort, prototypical monocyte derived inflammatory cytokines (IL-1 $\beta$, IL- 6 and TNF $\alpha$ ) were strongly intercorrelated, particularly in the unstimulated and TLR2stimulated groups, suggesting co-regulation of cytokine production. This is in keeping with a smaller study of young children ( $n=57$, age 5-96 months), which reported correlations between TNF $\alpha$ and IL-6 in whole blood following LPS stimulation (29). We also found moderate to strong correlations between the anti-inflammatory cytokines, IL-1RA and IL-10, following TLR stimulation, which is consistent with the complex co-regulation of cytokine responses and mechanisms for resolution of inflammation $(30,31)$.

As NCD prevalence is higher in males, we investigated sex differences in cytokine production in children. In adults, sex differences in cytokine responses are marked (29, 32-34), although this may partly reflect increased monocyte proportions (16). In our preschool cohort, only minor sex differences were evident for some cytokines following TLR stimulation; IL-12p70, IL-10, IL-6 and IL-1RA were modestly increased in boys, who also had higher monocyte proportions. Adjusting for the monocyte proportion attenuated the relationship of male sex and IL- 6 and IL-1RA, but not for IL12 p70 and IL-10 (data not shown). In addition, in sex-adjusted models, we found no association between BMI z-score or adiposity and cytokine levels, in contrast to reports from adults (35). This may reflect the relatively small number of children in this study with high BMI z-scores, or the relationship between $\mathrm{BMI}$ and innate immune responses may become evident later in life. This is also true for other potential confounders of NCDs, that have not been tested here. Although the adiposity measures were not correlated with innate immune cytokine levels, we noted that both GlycA and hsCRP were positively associated with BMI $\mathrm{z}$-score, in line with similar findings in adults and children (36). Both inflammatory markers were also positively associated with the proportion of granulocytes in these children, consistent with our previous findings from this cohort at 12 months (22) and reflective of generalised inflammatory status. Lastly, we found a correlation between levels of GlycA and CRP and season, which is in line with findings in adults (10).

The association between inflammatory status and cytokine levels in unstimulated and TLR-stimulated blood, which was independent of recent infection, has not previously been reported. As expected, the pro-inflammatory cytokines, IL- $1 \beta$ and IL- 6 were most strongly associated with GlycA and hsCRP, particularly in the unstimulated group. This association was not evident following TLR-4 stimulation, and interestingly the foldchange for these cytokines (stimulated/unstimulated) tended to decrease (data not shown); possibly reflecting the association between GlycA and hsCRP with anti-inflammatory cytokines following TLR2 and TLR4 stimulation.

This study has a number of strengths, including the relatively large sample size of young children with data on GlycA and hsCRP levels, as well as detailed cohort meta-data and immune measures. This allowed assessment of potential relationships and adjustment for important confounding variables, including the granulocyte and monocyte proportions, and sensitivity analysis for possible recent infection, which is common in this age group. All experiments were performed on fresh blood within 2 hours of collection, minimising process variation. We also acknowledge some limitations, including the cross-sectional design and the potential for type I errors, given the exploratory nature of the study and the multiple cytokines measured. In addition, it would have been valuable to have complete white blood cell counts, to expand our adjusted assays to lymphocyte counts in addition to monocytes and granulocytes. Although consideration was made for children with hsCRP levels above $5 \mathrm{ug} / \mathrm{ml}$, we did not have clinical or microbiological data on recent infections. Finally, future studies in later age groups should also adjust for other metabolic risk factors than BMI, such as lipid profile, glycemia, medication use or auto-immune diseases, which are not clearly apparent at the age of the children in our current study.

In summary, in healthy preschool children, levels of pro- and anti-inflammatory cytokines were correlated before and after TLR2 and TLR4 stimulation of whole blood samples. The inflammatory markers hsCRP and GlycA were positively correlated with innate immune cell activity. The findings support the concept that TLR activation contributes to the development of inflammatory NCD (12). Longitudinal studies from early life, with repeated, standardised measures of innate immune activation and circulating inflammatory markers may 
inform prediction of later NCD risk and highlight modifiable exposures and opportunities for early prevention.

\section{DATA AVAILABILITY STATEMENT}

The data generated for this study are part of the Barwon Infant Study and are available upon reasonable request. Requests to access the datasets should be directed to https://www. barwoninfantstudy.org.au/.

\section{ETHICS STATEMENT}

The studies involving human participants were reviewed and approved by Barwon Health Human Research Ethics Committee (HREC), project number 10/24. Written informed consent to participate in this study was provided by the participants' legal guardian/next of kin.

\section{AUTHOR CONTRIBUTIONS}

FC, CC, TM, and KF-C performed acquisition, analysis and interpretation of data. $\mathrm{SB}$ and $\mathrm{DB}$ contributed to the conceptualization and design of the study. FC, SB, and DB drafted the manuscript. All authors contributed to manuscript revision, read, and approved the submitted version.

\section{REFERENCES}

1. Daniels ME, Donilon TE, Bollyky TJ. The Emerging Global Health Crisis: Noncommunicable Diseases in Low- and Middle-Income Countries. SSRN Electron J (2016) 115:1-135. doi: 10.2139/ssrn.2685111

2. Lindblom R, Ververis K, Tortorella SM, Karagiannis TC. The Early Life Origin Theory in the Development of Cardiovascular Disease and Type 2 Diabetes. Mol Biol Rep (2015) 42:791-7. doi: 10.1007/s11033-014-3766-5

3. Coates MM, Kintu A, Gupta N, Wroe EB, Adler AJ, Kwan GF, et al. Burden of Non-Communicable Diseases From Infectious Causes in 2017: A Modelling Study. Lancet Glob Heal (2020) 8:e1489-98. doi: 10.1016/S2214-109X(20) 30358-2

4. Camps J, García-Heredia A. Introduction: Oxidation and Inflammation, a Molecular Link Between Non-Communicable Diseases. Adv Exp Med Biol (2014) 824:1-4. doi: 10.1007/978-3-319-07320-0_1

5. Furman D, Campisi J, Verdin E, Carrera-Bastos P, Targ S, Franceschi C, et al. Chronic Inflammation in the Etiology of Disease Across the Life Span. Nat Med (2019) 25:1822-32. doi: 10.1038/s41591-019-0675-0

6. Coates BM, Staricha KL, Wiese KM, Ridge KM. Influenza a Virus Infection, Innate Immunity, and Childhood. JAMA Pediatr (2015) 169:956-63. doi: 10.1001/jamapediatrics.2015.1387

7. Simon AK, Hollander GA, McMichael A. Evolution of the Immune System in Humans From Infancy to Old Age. Proc R Soc B Biol Sci (2015) 282:20143085. doi: $10.1098 / \mathrm{rspb} .2014 .3085$

8. Öner D, Drysdale SB, McPherson C, Lin GL, Janet S, Broad J, et al. Biomarkers for Disease Severity in Children Infected With Respiratory Syncytial Virus: A Systematic Literature Review. J Infect Dis (2020) 222: S648-57. doi: 10.1093/INFDIS/JIAA208

9. Burgner D, Liu R, Wake M, Uiterwaal CSP. Do Childhood Infections Contribute to Adult Cardiometabolic Diseases? Pediatr Infect Dis J (2015) 34:1253-5. doi: 10.1097/INF.0000000000000882

10. ter Horst R, Jaeger M, Smeekens SP, Oosting M, Swertz MA, Li Y, et al. Host and Environmental Factors Influencing Individual Human

\section{FUNDING}

The initial establishment work and infrastructure for the BIS was supported by the Murdoch Children's Research Institute and Barwon Health. Deakin University is now a partner organization and has provided funding and infrastructure. Funding for this study was provided by the National Health and Medical Research Council of Australia, NHMRC (1030701). SB is supported by the Dutch Scientific Organisation (452173113) and the Dutch Heart Foundation (2018T028). DB is supported by a NHMRC Investigator Grant (1175744). Research at the Murdoch Children's Research Institute is supported by the Victorian Government's Operational Infrastructure Program. The funding bodies did not play any role in the study.

\section{ACKNOWLEDGMENTS}

We would like to acknowledge the participation and commitment of the families in the Barwon Infant Study (BIS).

\section{SUPPLEMENTARY MATERIAL}

The Supplementary Material for this article can be found online at: https://www.frontiersin.org/articles/10.3389/fimmu.2021.830049/ full\#supplementary-material

Cytokine Responses. Cell (2016) 167:1111-24.e13. doi: 10.1016/ j.cell.2016.10.018

11. Kawasaki T, Kawai T. Toll-Like Receptor Signaling Pathways. Front Immunol (2014) 5:461. doi: 10.3389/fimmu.2014.00461

12. Wong SK, Chin K-Y, Ima-Nirwana S. Toll-Like Receptor as a Molecular Link Between Metabolic Syndrome and Inflammation: A Review. Curr Drug Targets (2019) 20:1264-80. doi: 10.2174/1389450120666190405172524

13. Shaw AC, Panda A, Joshi SR, Qian F, Allore HG, Montgomery RR. Dysregulation of Human Toll-Like Receptor Function in Aging. Ageing Res Rev (2011) 10:346-53. doi: 10.1016/j.arr.2010.10.007

14. Ziakas PD, Prodromou ML, El Khoury J, Zintzaras E, Mylonakis E. The Role of TLR4 896 a $>$ G and 1196 C>T in Susceptibility to Infections: A Review and Meta-Analysis of Genetic Association Studies. PloS One (2013) 8:e81047. doi: 10.1371/journal.pone.0081047

15. Miller JE, Goldacre R, Moore HC, Zeltzer J, Knight M, Morris C, et al. Mode of Birth and Risk of Infection-Related Hospitalisation in Childhood: A Population Cohort Study of 7.17 Million Births From 4 High-Income Countries. PloS Med (2020) 17 :e1003429. doi: 10.1371/journal.pmed.1003429

16. Beenakker KGM, Westendorp RGJ, De Craen AJM, Chen S, Raz Y, Ballieux $\mathrm{BEPB}$, et al. Men Have a Stronger Monocyte-Derived Cytokine Production Response Upon Stimulation With the Gram-Negative Stimulus Lipopolysaccharide Than Women: A Pooled Analysis Including 15 Study Populations. J Innate Immun (2020) 12:142-53. doi: 10.1159/000499840

17. Lefevre N, Noyon B, Biarent D, Corazza F, Duchateau J, Casimir G. SEX DIFFERENCES in INFLAMMATORY RESPONSE and ACID'base BALANCE in PREPUBERTAL CHILDREN With SEVERE SEPSIS. Shock (2017) 47:422-8. doi: 10.1097/SHK.0000000000000773

18. Fuertes-Martín R, Correig X, Vallvé JC, Amigó N. Human Serum/Plasma Glycoprotein Analysis Bylh-NMR, an Emerging Method of Inflammatory Assessment. J Clin Med (2020) 9:354. doi: 10.3390/jcm9020354

19. Kettunen J, Ritchie SC, Anufrieva O, Lyytikäinen LP, Hernesniemi J, Karhunen PJ, et al. Biomarker Glycoprotein Acetyls Is Associated With the Risk of a Wide Spectrum of Incident Diseases and Stratifies Mortality Risk in 
Angiography Patients. Circ Genomic Precis Med (2018) 11:e002234. doi: 10.1161/CIRCGEN.118.002234

20. Vuillermin P, Saffery R, Allen KJ, Carlin JB, Tang MLK, Ranganathan S, et al. Cohort Profile: The Barwon Infant Study. Int J Epidemiol (2015) 44:1148-60. doi: 10.1093/ije/dyv026

21. Collier FM, Tang MLK, Martino D, Saffery R, Carlin JB, Jachno K, et al. The Ontogeny of Naive and Regulatory CD4+ T-Cell Subsets During the First Postnatal Year: A Cohort Study. Clin Transl Immunol (2015) 4:e34. doi: $10.1038 /$ cti.2015.2

22. Collier F, Ellul S, Juonala M, Ponsonby AL, Vuillermin P, Saffery R, et al. Glycoprotein Acetyls (GlycA) at 12 Months Are Associated With HighSensitivity C-Reactive Protein and Early Life Inflammatory Immune Measures. Pediatr Res (2019) 85:584-5. doi: 10.1038/s41390-019-0307-x

23. Lycett K, Juonala M, Magnussen CG, Norrish D, Mensah FK, Liu R, et al. Body Mass Index From Early to Late Childhood and Cardiometabolic Measurements at 11 to 12 Years. Pediatrics (2020) 146:e20193666. doi: 10.1542/peds.2019-3666

24. Lemiengre MB, Verbakel JY, Colman R, Van Roy K, De Burghgraeve T, Buntinx F, et al. Point-Of-Care CRP Matters: Normal CRP Levels Reduce Immediate Antibiotic Prescribing for Acutely Ill Children in Primary Care: A Cluster Randomized Controlled Trial. Scand J Prim Health Care (2018) 36:423-36. doi: 10.1080/02813432.2018.1529900

25. Verbakel JY, Lemiengre MB, De Burghgraeve T, De Sutter A, Aertgeerts B, Shinkins B, et al. Should All Acutely Ill Children in Primary Care be Tested With Point-of-Care CRP: A Cluster Randomised Trial. BMC Med (2016) 14:131. doi: 10.1186/s12916-016-0679-2

26. Burgner DP, Cooper MN, Moore HC, Stanley FJ, Thompson PL, De Klerk $\mathrm{NH}$, et al. Childhood Hospitalisation With Infection and Cardiovascular Disease in Early-Mid Adulthood: A Longitudinal Population-Based Study. PloS One (2015) 10:e0125342. doi: 10.1371/journal.pone.0125342

27. Burgner DP, Sabin MA, Magnussen CG, Cheung M, Kähönen $M$, Lehtimäki T, et al. Infection-Related Hospitalization in Childhood and Adult Metabolic Outcomes. Pediatrics (2015) 136:e554-62. doi: 10.1542/ peds.2015-0825

28. Lefèvre N, Corazza F, Duchateau J, Desir J, Casimir G. Sex Differences in Inflammatory Cytokines and CD99 Expression Following In Vitro Lipopolysaccharide Stimulation. Shock (2012) 38:37-42. doi: 10.1097/ SHK.0b013e3182571e46

29. Casimir GJ, Heldenbergh F, Hanssens L, Mulier S, Heinrichs C, Lefevre N, et al. Gender Differences and Inflammation: An In Vitro Model of Blood Cells Stimulation in Prepubescent Children. J Inflamm (2010) 7:28. doi: 10.1186/ 1476-9255-7-28

30. Schmitz ML, Weber A, Roxlau T, Gaestel M, Kracht M. Signal Integration, Crosstalk Mechanisms and Networks in the Function of Inflammatory
Cytokines. Biochim Biophys Acta - Mol Cell Res (2011) 1813:2165-75. doi: 10.1016/j.bbamcr.2011.06.019

31. Wolf AM, Wolf D, Rumpold H, Enrich B, Tilg H. Adiponectin Induces the AntiInflammatory Cytokines IL-10 and IL-1RA in Human Leukocytes. Biochem Biophys Res Commun (2004) 323:630-5. doi: 10.1016/j.bbrc.2004.08.145

32. Von Aulock S, Deininger S, Draing C, Gueinzius K, Dehus O, Hermann C. Gender Difference in Cytokine Secretion on Immune Stimulation With LPS and LTA. J Interf Cytokine Res (2006) 26:887-92. doi: 10.1089/ jir.2006.26.887

33. Temple S EL, Pham K, Glendenning P, Phillips M, Waterer GW. Endotoxin Induced TNF and IL-10 mRNA Production Is Higher in Male Than Female Donors: Correlation With Elevated Expression of TLR4. Cell Immunol (2008) 251:69-71. doi: 10.1016/j.cellimm.2008.04.013

34. Wirtz PH, Von Känel R, Rohleder N, Fischer JE. Monocyte Proinflammatory Cytokine Release Is Higher and Glucocorticoid Sensitivity Is Lower in Middle Aged Men Than in Women Independent of Cardiovascular Risk Factors. Heart (2004) 90:853-8. doi: 10.1136/hrt.2002.003426

35. Scholtes VPW, Versteeg D, De Vries JPPM, Hoefer IE, Schoneveld AH, Stella PR, et al. Toll-Like Receptor 2 and 4 Stimulation Elicits an Enhanced Inflammatory Response in Human Obese Patients With Atherosclerosis. Clin Sci (2011) 121:205-14. doi: 10.1042/CS20100601

36. Bartlett DB, Slentz CA, Connelly MA, Piner LW, Willis LH, Bateman LA, et al. Association of the Composite Inflammatory Biomarker Glyca, With ExerciseInduced Changes in Body Habitus in Men and Women With Prediabetes. Oxid Med Cell Longev (2017) 2017:3608287. doi: 10.1155/2017/5608287

Conflict of Interest: The authors declare that the research was conducted in the absence of any commercial or financial relationships that could be construed as a potential conflict of interest.

Publisher's Note: All claims expressed in this article are solely those of the authors and do not necessarily represent those of their affiliated organizations, or those of the publisher, the editors and the reviewers. Any product that may be evaluated in this article, or claim that may be made by its manufacturer, is not guaranteed or endorsed by the publisher.

Copyright (c) 2022 Collier, Chau, Mansell, Faye-Chauhan, Vuillermin, Ponsonby, Saffery, Tang, O'Hely, Carlin, Gray, Bekkering, Burgner and the Barwon Infant Study Investigator Group. This is an open-access article distributed under the terms of the Creative Commons Attribution License (CC BY). The use, distribution or reproduction in other forums is permitted, provided the original author(s) and the copyright owner(s) are credited and that the original publication in this journal is cited, in accordance with accepted academic practice. No use, distribution or reproduction is permitted which does not comply with these terms. 